\title{
The use of evolutionary algorithms for designing an optimum structure of a geodesic measurement and control network
}

\author{
Maria Mrówczyńska ${ }^{1, *}$, Jacek Sztubecki ${ }^{2}$ \\ ${ }^{1}$ University of Zielona Góra, Faculty of Civil Engineering, Architecture and Environmental Engineering, ul. Prof. Z. Szafrana 1, \\ 65-516 Zielona Góra, Poland \\ ${ }^{2}$ University of Science and Technology, Faculty of Civil and Environmental Engineering and Architecture, ul. Prof. S. Kaliskiego 7 , \\ 85-796 Bydgoszcz, Poland
}

\begin{abstract}
The paper presents an attempt to determine an optimum structure of a geodesic measurement and control network used for geodesic monitoring to determine horizontal displacements of buildings. In geodesy, horizontal networks can be used to determine terrain deformations as well as displacements of engineering structures (dams, water reservoirs, open-cast mines). The network subjected to analysis is a directional network. In order to find a correct solution, its structure should include so-called supernumerary observations. An adequate number of observations should be carried out in the network to obtain a solution with reliable values of horizontal displacements. Moreover, the way in which the observations are carried out and their number should make it possible to show changes taking place in the object and meet the economic criteria of geodesic measurements. In order to optimize the structure of a geodesic measurement and control network, information entropy and evolutionary algorithms are used in the paper. Information entropy is a logarithmic measure of probability, and an optimum number of observations carried out in the network depends on the increment of the content of information in the observation system. Evolutionary algorithms were developed in the 1980s, and they are currently very popular and widely used. Their main principle is based on the evolution or behaviour of the best adapted individuals in subsequent computational cycles.
\end{abstract}

\section{Introduction}

According to current regulations [1] in order to ensure safety during the construction of a building and its operation, it is necessary to carry out geodesic measurements to determine displacements of the object and its foundation as well as its deformations, and to identify the impact of the object on neighbouring objects. Displacement or deformation monitoring may be required for both existing and new buildings, adjacent buildings, as well as for ground or groundwater (water table). The determination of displacements and deformations, both with classical methods, GNSS methods and by laser scanning, should be preceded by an analysis of the existing field conditions (also with the participation of GIS tools [2]) and selection of a suitable structure for the measurement and control network set up on the object [3, $4,5]$. In the case of construction work, which requires displacement or deformation monitoring, the following should be specified in particular:

- the measurement method, including the measurement equipment that will be used for determining displacements,

- the frequency of measurements and assessment of their accuracy,

- the method for presenting data results,
- the location of observation stands (horizontally and vertically),

- the frequency of checking the stability of the observation stands and reference points during the construction work and after its completion.

The inspection of changes in the position of elements of engineering objects during their construction or operation may be single or periodical. A single measurement provides information about the current state of the object, which can be compared with the theoretical model. In the case of periodical measurements, it is very important to determine their frequency and duration. The frequency, i.e. the time interval between two successive periodical measurements, is selected in such a way that the expected change in displacements is within $2 M_{p} \div 0,5 M p$, where $M_{p}$ is the boundary error. However, the duration of a periodical measurement should be so short that the expected change in displacement is smaller than the mean error of the measurement $m_{p}$. In this context, it is important to plan the structure of the measurement and control network in such a way that it ensures that changes in the object are recorded and that it is possible to carry out measurements at the right time [6]. The formulation of the problem of optimizing the structure of a measurement and control network presented in the paper is based on minimization of the objective function, defined as information entropy that transmits a specific

* Corresponding author: m.mrowczynska@ib.uz.zgora.pl 
amount of information on the current state of the observation system [7]. The minimization of the objective function was carried out in the paper on the basis of a solution using evolutionary techniques.

Evolutionary algorithms can be used in technology, economics and other fields of knowledge, wherever there is a need to search for better and better solutions and to optimize these solutions [8]. In theory, optimization is a search for the best solution, which in practice usually means searching for a better solution than the one previously known. Optimization tasks can be solved with analytical or numerical methods that search for a minimum (or maximum) of the objective function in a set of acceptable solutions. However, in each case we face the problem of selecting an optimization algorithm that would lead to solving the task at the lowest possible cost.

\section{Methods}

Evolutionary algorithms are a wide family of algorithms that are based on the principle of the process of evolution, i.e. the survival of the best adapted individuals. Evolutionary algorithms include: genetic algorithms, genetic programming, evolutionary strategies and evolutionary programmes $[9,10]$. Solving problems with the use of evolutionary algorithms consists in searching for a potential solution through transformation sequences with the use of genetic operators [11]. The most common genetic operators include selection, cross-breeding and mutation. Besides the ones mentioned above, it is also possible to use dominance, inversion, detection, segregation or translocation. The objective of selection is to create a new $(k+1)$ generation of individuals. Crossbreeding (Fig. 1) takes place in two steps and makes it possible to create new individuals by combining several individuals by the cross-breeding operator. In the first step, the individuals that will be crossbred are selected from the current population, and in the second step, crossbreeding occurs, i.e. an exchange of some characters in the binary chains of the selected individuals.

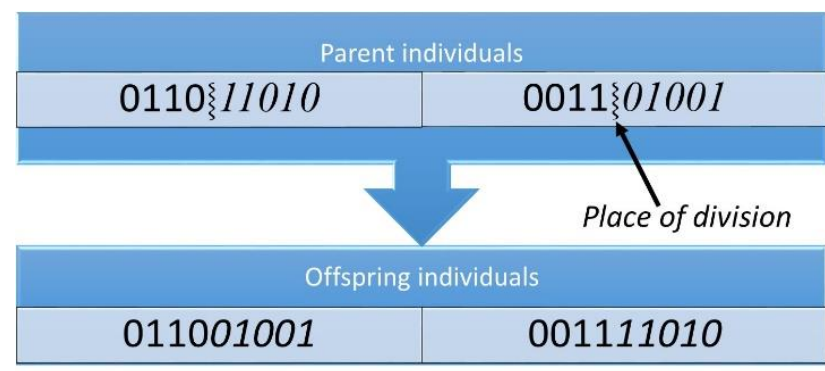

Fig. 1. Cross-breeding in binary coding (prepared by the Authors).

The objective of mutation is to create new individuals by changing characters in the binary chain of a particular individual (Fig. 2). Of these two operators, cross-breeding is more important because mutation is a random walk in the space of acceptable solutions. However, it should always be present in the evolutionary algorithm because it can prevent an irreversible loss of information that can occur during cross-breeding and selection.

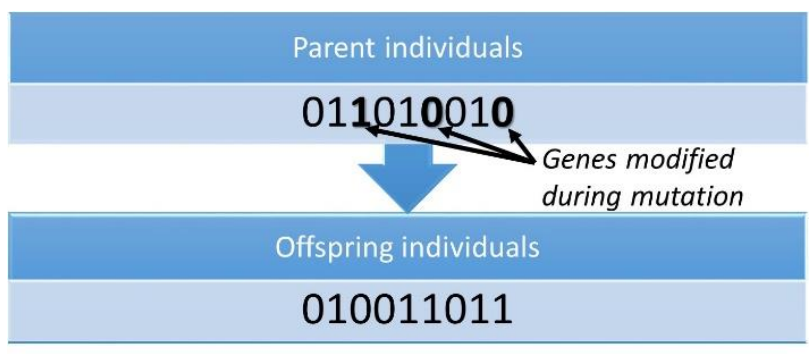

Fig. 2. Mutation in binary coding (prepared by the Authors).

Evolutionary algorithms belong to probabilistic algorithms that manage a certain population of individuals $P(k)$ in the $k$-th generation. An algorithm operates on the principle that a certain number of iterations (called generations) are performed until the stop criterion is reached. In each iteration, the specimens are transformed with the use of the previously mentioned genetic operators. In this way a new generation $(k+1)$ is formed, which consists of the best adapted individuals. The measure of adaptation of a single individual $n$ is the adaptation function $\operatorname{ch}(n)$ whose value determines the survival of a particular individual. A diagram of the structure of the evolutionary algorithm is presented in Figure 3.

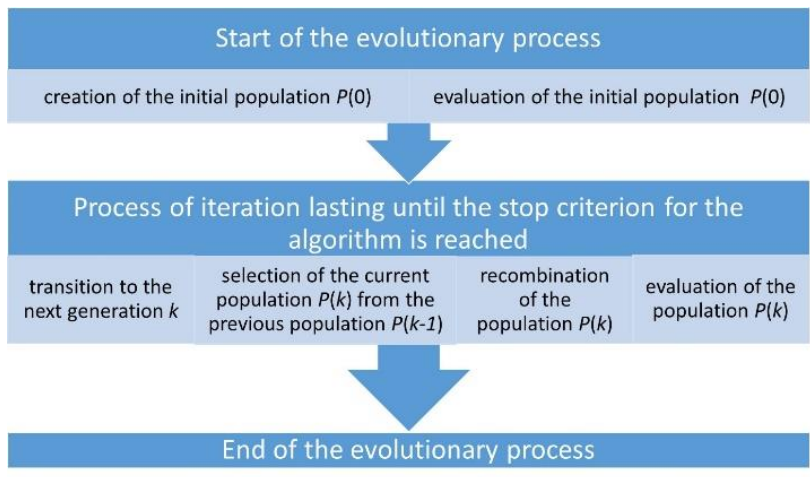

Fig. 3. A diagram of the operation of an evolutionary algorithm (prepared by the Authors).

A number of evolutionary algorithms can be formulated to solve the problems discussed in the paper. However, all of them have a common feature - the population undergoes certain transformations, which makes it possible to select the best adapted individuals. Every evolutionary algorithm has to include the following elements [8]:

- a representation of the problem in genetic form; Optimization of the objective function $f\left(x_{1}, x_{2}, \ldots, x_{n}\right)$ requires that the variable $x_{i}$ should be represented by a binary chain with an adequate length. In order to ensure that the solution has the required accuracy, for each variable $x_{i}$ the inequality (1) has to be satisfied: 


$$
\left(t_{i}-r_{i}\right) \cdot 10^{p i} \leq 2^{l i}-1
$$

where:

$r_{i}, t_{i}$ - the upper and the lower boundaries of the domain of the variable $x_{i}$,

$p_{i}$ - the required accuracy of the variable $x_{i}$,

$l_{i}$ - the length of the binary chain of the variable $x_{i}$ expressed in bits,

- the method for creating the initial population of possible solutions,

- the evaluation function that makes it possible to evaluate the solution in terms of the adaptation of particular individuals,

- the genetic operators changing the composition of the population,

- the parameters used by evolutionary algorithms that make it possible to assess the correctness of the proposed solution.

While using evolutionary algorithms for solving optimization problems, and bearing in mind that a large number of such algorithms can be formulated as mentioned before, it is necessary to look for one that will make it possible to find a solution at the lowest cost, and for which the solution will meet the expected accuracy characteristics. Moreover, the algorithm should be robust, which means that it should be able to generate solutions whose position will be as little dependent as possible on the state of the initial population. If these conditions are met, it will be possible to obtain similar final results with a number of independent executions of the algorithm [11].

\section{Results}

For the analysis, whose task was to determine an optimum network structure, part of a geodesic measurement and control network consisting of 12 points was used, where 78 directions were observed (Fig. 4).

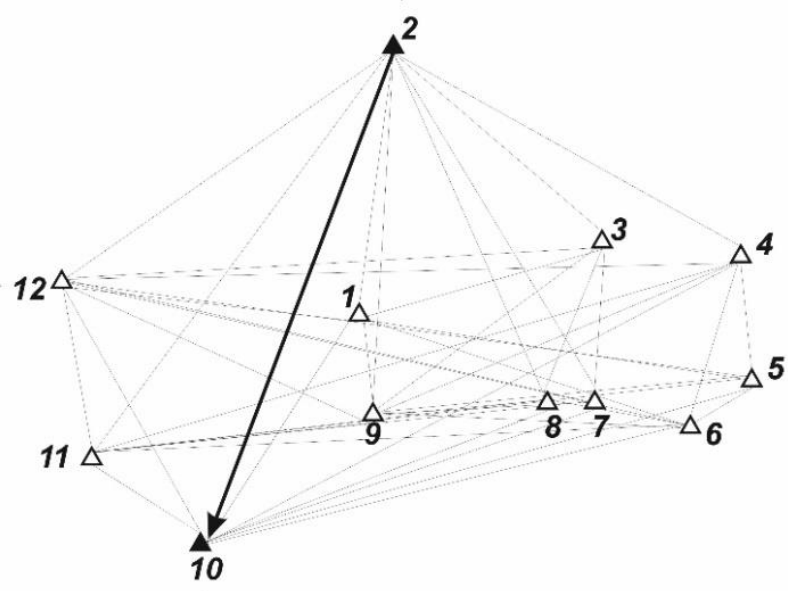

Fig. 4. The structure of a horizontal measurement and control network (prepared by the authors for selected observations).

The number of directions observed exceeded more than twice the number of necessary observations involved in the process of solving and adjusting the network. The network designed in this way and the observations carried out ensured that the displacements had been correctly determined. In order to perform the analysis, the network was pre-prepared as follows:

- the network was adjusted with minimum restrictions on the degrees of freedom, assuming that points 2 and 10 were fixed, and with an a priori mean error of the direction measurement $m_{d i r}= \pm 5^{\mathrm{cc}}$,

- the adjustment was carried out with the strict method using on the postulate of the least square method $[v v]=$ min, where $v$-is the adjustment to the observation,

- the mean network error after the adjustment was obtained as $m_{0}=1,12$,

- three outliers were identified and eliminated, for which the numerical ratio between the adjustment $v$ and the adjustment error $m_{v}$ met the condition $\frac{v}{m_{v}} \geq 2$.

In the next step, according to the formula (2), the entropy of the observation system $H_{\widehat{\mathbf{x}}}(\mathbf{x})$ was determined in the aspect of the entropy of the vector of parameters $\hat{\mathbf{x}}$ in relation to the true value $\mathbf{x}[12]$.

$$
H_{\hat{\mathbf{x}}}(\mathbf{x})=\log _{2}\left[\frac{g(m, n) m_{d i r} \Pi_{j=1}^{n} \sqrt{\sum_{i=1}^{m} a_{i j}^{2}}}{\prod_{j=1}^{n} \varepsilon_{j} \operatorname{det}\left(\mathbf{A}^{\mathbf{T}} \mathbf{A}\right)}\right]
$$

where:

$m_{\text {dir. }}$ - the a priori mean error of a single observation (direction) $\left[{ }^{\mathrm{cc}}\right]$,

$\mathbf{A}$ - the matrix of the system of normal equations, $\varepsilon_{j}=m_{0} \sqrt{\frac{\delta_{0}}{\gamma_{j}}}-$ the sensitivity of the system along a particular axis of coordinates, and: $\delta_{0}$ - the non-centrality parameter, $\gamma_{j}$-the minimum eigenvalue of the product of the matrixes $\mathbf{A}^{\mathrm{T}} \mathbf{A}$.

While optimizing the structure of the measurement network, it was also necessary to determine the contribution of a single supernumerary observation to the overall state of the whole network, this content of information was determined on the basis of the following formula [13]:

$$
h=\log _{2} \frac{g(m, n)\left\{1+\operatorname{trace}\left[\left(\mathbf{A}^{\mathrm{T}} \mathbf{A}\right)^{-1}\left(b^{\mathrm{T}} b\right)\right]\right\}}{g(m+1, n) \prod_{j=1}^{n} \sqrt{1+\frac{a_{m+1, j}^{2}}{\sum_{i=1}^{m} a_{i j}^{2}}}}
$$

Particular symbols in the formula (3) denote:

$b$ - the subsequent line of the matrix $\mathbf{A}$,

$g(m, n)$ - the value determined on the basis of the standard deviation of the probability density function and the value of the Euler function.

The content of information in particular observations (Fig. 5) varies depending on the fixed points set up during the network adjustment process, but the content of information in the whole system is approximately constant. 


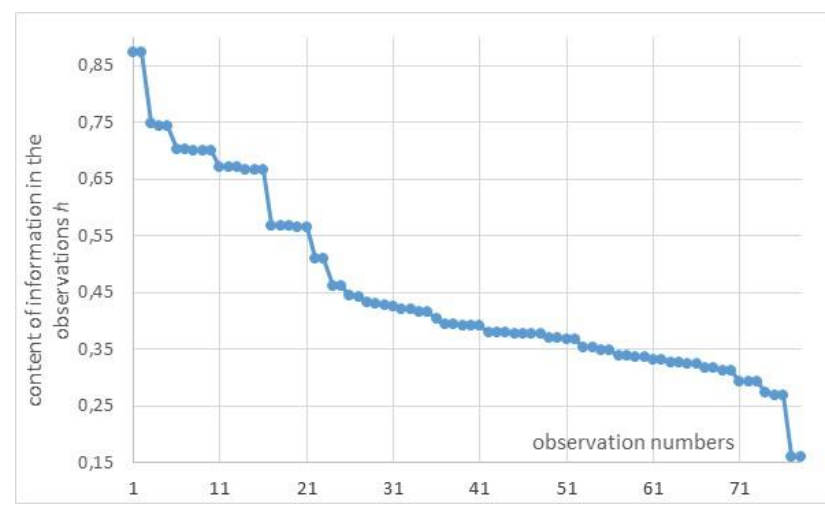

Fig. 5. The content of information in particular observations (prepared by the Authors for the observations measured in the network).

The content of information in the whole system, after eliminating the outliers (for 75 observations), is $\sum h=$ 17,2509 , and the entropy of the vector of parameters is $H_{\hat{\mathbf{x}}}(\mathbf{x})=19.7254$. If we took into account all directional observations possible to carry out in the measurement network, then the entropy would be $H_{\hat{\mathbf{x}}}(\mathbf{x})=22.8431$. The most important task in terms of correctness of the values of horizontal displacements and the economics of measurements is to properly design the structure of the measurement and control network. An evolutionary algorithm based on the concepts and solutions given in the papers $[14,15]$ was used to design an optimum structure. The process of designing an optimum network structure consisted in determining a set of necessary angular observations. The resulting set of observations has to be characterized by supernumerary observations and a minimum value of the entropy of the vector of parameters $H_{\hat{\mathbf{x}}}(\mathbf{x})$ dependent both on the number of observations and their configuration. A general diagram of the algorithm used is presented in Fig. 6 and discussed below.

Determination of genetic parameters (the size of the population, probability of cross-breeding and mutations, the stop criterion). 5

Creation of the initial population. Each individual should be a random permutation of angular observations assigned to precisely specified points.

Execution of the operation of cross-breeding.

Execution of the operation of mutation.

Calculation of the adaptation function for particular individuals.

Creation of a new population.

Use of the elitist strategy (removal of one observation and insertion of the best one from the previous population).

Checking the stop criterion for the algorithm.

Fig. 6. A solution to the problem of geodesic network optimization with the use of an evolutionary algorithm (prepared by the Authors).

In an evolutionary algorithm, each individual consists of a set of genes, whereas each gene is an ordered pair in the form of a controlled point (observation stand) and an angular observation defined by specifying the direction of its execution. The structure of the chromosome is presented in Fig. 7.
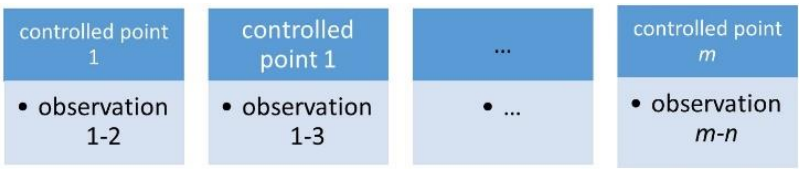

Fig. 7. The structure of the chromosome - the coding method (prepared by the Authors).

In the problem of optimization of the geodesic network structure solved in this paper, the random procedure for creating the initial population cannot be used because of the limitations that require the occurrence of supernumerary observations in the network. For this reason, the observations of directions with the largest content of information were adopted as the initial population. The objective function was defined as the entropy of the vector of parameters defined by the formula (2). During the operation of the evolutionary algorithm, selection, cross-breeding and mutation operators were used (Fig. 6).

The selection was carried out with a method based on the roulette wheel combined with the elitist strategy. This approach prevents elimination of the best adapted individuals, which could occur as a result of random sampling if the roulette wheel method were the only one used.

The function described by the formula (4) was adopted as the adaptation function for each individual (observation of the direction) in the population.

$$
\operatorname{ch}\left(H_{k}\right)=\frac{H_{\max }^{0}-H_{k}}{H_{\max }^{0}-H_{\min }^{0}}
$$

where:

$H_{\max }^{0}$ - the maximum value of the objective function in the initial population,

$H_{m i n}^{0}$ - the minimum value of the objective function in the initial population,

$H_{k}$ - the value of the objective function in the current population.

The calculations were carried out with the use of source codes prepared with the MATLAB software [https://www.mathworks.com], and the following parameters were adopted:

- the size of the initial population as the minimum number of observations of directions necessary to carry out $N=25$,

- the stop criterion for the algorithm as the maximum number of iterations carried out $k=1000$,

- the probability of cross-breeding $p_{k}=0,5$,

- the probability of mutation $p_{m}=0,05$.

As a result of the operation of the algorithm minimizing the objective function (2), it was found that the number of observations of directions that should be carried out in the network was 52 . The evolutionary algorithm was initiated a number of times in order to obtain the best solution and verify it. A detailed compilation of the results in the form of the values of the information entropy of the observation system dependent on the number of planned observations is shown in Table 1. 
Table 1. The values of the entropy of the vector of parameters (of the objective function) in relation to the adopted number of observations

\begin{tabular}{|c|c|c|}
\hline No. & $\begin{array}{c}\text { Value of the } \\
\text { objective } \\
\text { function } H_{\hat{\mathbf{x}}}(\mathbf{x})\end{array}$ & Number of observations \\
\hline 1 & 21.4332 & $\begin{array}{c}\mathbf{2 5} \text { observations necessary to } \\
\text { solve the network }\end{array}$ \\
\hline 2 & 19.0205 & $\begin{array}{c}\mathbf{5 2} \text { observations determined as a } \\
\text { result of the operation of the } \\
\text { evolutionary algorithm }\end{array}$ \\
\hline 3 & 19.7254 & $\begin{array}{c}\mathbf{7 5} \text { observations that were } \\
\text { adopted for further calculations } \\
\text { after eliminating the outliers } \\
\text { (initially 78) }\end{array}$ \\
\hline 4 & 22.8431 & $\begin{array}{c}\mathbf{1 3 2} \text { observations - all possible to } \\
\text { carry out in the network }\end{array}$ \\
\hline
\end{tabular}

It is worth noting that the use of evolutionary algorithms to solve the problem of optimization of the geodesic network structure makes it possible to both determine an optimum number of observations and to show which observations (the best adapted individuals) should be carried out in the network because of their content of information.

\section{Conclusions}

The article presents one of the possible methods for optimizing the horizontal structure of a geodesic measurement and control network used for determining displacements and deformations of engineering structures. The optimization was carried out with the use of evolutionary algorithms, and the entropy of the vector of parameters was adopted as the objective function. This solution makes it possible to determine the content of information in individual observations, which influences the selection of individuals of the initial population. The use of the evolutionary approach ensures appropriate selection of the number of observations and their configuration in relation to one another, which makes it possible to increase the economic efficiency of the work done and to ensure favourable accuracy characteristics. This is important both in the case of classic geodesic measurements, GNSS measurements, and measurements carried out with the use of laser scanning. The numerical experiments show that evolutionary algorithms can be used as an effective computational tool for optimization of measurement structures, and they can also be used to build mathematical models of phenomena and processes observed.

However, it is worth emphasizing that a characteristic feature of evolutionary algorithms (as well as all nondeterministic methods) is that with the same values of parameters and the initial population, it is possible to notice different results obtained from different algorithm executions. The reason for this is the use of selection and genetic operators. Therefore, it is necessary to carry out the procedure of repeated algorithm executions, which will allow the algorithm to leave the area of local attraction by the maximum of the adaptation function.

\section{References}

1. Directive of the Minister of Spatial Planning and Construction of 21 February 1995 on the type and range of geodesic and cartographic analyses and geodesic tasks in the construction industry Journal of Laws of the Republic of Poland 1995 No. 25 item 133

2. Sz. Szewrański, J. Kazak, M. Sylla, M. Świąder, Spatial Data Analysis with the Use of ArcGIS and Tableau Systems, In: Ivan I., Singleton A., Horák J., Inspektor T. (eds) The Rise of Big Spatial Data. Lecture Notes in Geoinformation and Cartography. Springer, Cham pp 337-349 (2017)

3. R. Kadaj, Modele, metody i algorytmy obliczeniowe sieci kinematycznych $w$ geodezyjnych pomiarach przemieszczeń $i$ odksztatceń obiektów (Wydawnictwo Akademii Rolniczej, Kraków 1998)

4. J. Zaczek-Peplińska, M. Kowalska, terrestrial laser scanning in monitoring hydrotechnical objects, Journal of Ecological Engineering 17, 4 pp. 120-128 (2016)

5. K. Karsznia, The assessment of modern photogrammetric surveying methods in road works applications, Conference: 17th International Multidisciplinary Scientific Geoconference SGEM 17 pp. 271-278 (2017)

6. I. Skrzypczak, W. Kokoszka, J. Kogut, G. Oleniacz, Methods of Measuring and Mapping of Landslide Areas, World Multidisciplinary Earth Sciences Symposium 2017, Book Series: IOP Conference Series-Earth and Environmental Science 95 (2017)

7. M. Mrówczynska, Analysis of the horizontal structure of a measurement and control geodetic network based on entropy, Geodesy and Cartography 62, 1 pp. 23-31 (2013)

8. J. Patalas-Maliszewska, I.Krebs, The optimization of the process of knowledge worker selection using a game theory approach, Methods and Models i Automation and Robotics pp. 820-823 (2014)

9. A. Helt, M. Parol, P. Piotrowski, Metody sztucznej inteligencji. Przykłady zastosowań w elektrotechnice (Oficyna Wydawnicza Politechniki Warszawskiej, Warsaw 2012)

10. G. Lindfield, J. Penny, Chapter 2-Evolutionary Algorithms, Introduction to Nature-Inspired Optimization pp. 19-47 (Academic Press 2017)

11. J. Arabas, Wyktady z algorytmów ewolucyjnych (Wydawnictwo Naukowo-Techniczne, Warsaw 2001)

12. E. Nowak, W. Pruszyński, Analysis of horizontal engineering survey networs theory and worked examples, (Warsaw 1990)

13. Ju. Neuman, $K$ analizu geodeziczeskich postrojenii, Izwestia wuzow, Geodezja i Aerofotosiomka 4 (1965) 
14. R. Cheng, M. Gen, T. Tozawa, Fuzzy Vehicle Routing and Scheduling Oriblem Using Genetic Algorithms in F. Herrera F. and J. Verdegay Genetic Algorithms and Soft Computing (Springer-Veralg, 1996)

15. M. Gen, R. Cheng, Genetic Algorithms \& Engineering Design (John Wiley \& Sons, Inc., New York 1997) 